九州大学学術情報リポジトリ

Kyushu University Institutional Repository

\title{
Numerical study on transient harbor oscillations induced by solitary waves
}

Gao, Junliang

School of Naval Architecture and Ocean Engineering, Jiangsu University of Science and Technology

Ji, Chunyan

School of Naval Architecture and Ocean Engineering, Jiangsu University of Science and Technology

Liu, Yingyi

Research Institute for Applied Mechanics, Kyushu University

Gaidai, 0 leg

School of Naval Architecture and Ocean Engineering, Jiangsu University of Science and Technology

他

http://hdl. handle. net/2324/4055212

出版情報 : Ocean Engineering. 126，pp.467-480，2016-11-01. Elsevier バージョン：

権利関係: Creative Commons Attribution NonCommercial NoDerivatives 4.0 International 
Numerical study on transient harbor oscillations induced by solitary waves

Junliang Gao ${ }^{\text {a b, c, }{ }^{*} \text {, Chunyan Ji }}{ }^{\text {a }}$, Yingyi Liu ${ }^{\text {d, Oleg Gaidai }}{ }^{\text {a }}$, Xiaojian Ma ${ }^{a}$, Zhen Liu ${ }^{\text {a }}$

a School of Naval Architecture and Ocean Engineering, Jiangsu University of Science and Technology, Zhenjiang 212003, China

b Jiangsu Key Laboratory of Coast Ocean Resources Development and Environment Security, Hohai University, Nanjing 210098, China

c State Key Laboratory of Coastal and Offshore Engineering, Dalian University of Technology, Dalian 116024, China

d Research Institute for Applied Mechanics, Kyushu University, Kasuga, Fukuoka 816-8580, Japan

\begin{abstract}
:
The main purpose of this article is to systematically investigate the influence of the variation of the incident wave height and the bottom profile inside an elongated rectangular harbor on relevant physical phenomena involved in transient harbor oscillations induced by normal-incident solitary waves. These phenomena include wave height evolution, oscillation amplification, total wave energy and relative wave energy distribution inside the harbor. A series of numerical experiments are carried out using the FUNWAVE 2.0 model. Results show that the height evolution of the incident wave during the shoaling process inside the harbor coincides well with Green's law. When the wave nonlinearity is relatively weak, the maximum oscillation inside the harbor can be regarded as increasing linearly with the incident solitary wave height; while as the wave nonlinearity becomes strong, the amplification factor of the incident solitary wave increases gradually with the wave nonlinearity. The total wave energy trapped in the harbor depends on both the mean water depth and the bottom profile. The relative wave energy distribution inside the harbor is greatly affected by the incident solitary wave height; however, the variation of the bottom profile inside the harbor has a negligible effect on it.
\end{abstract}

\title{
Key words:
}

Harbor resonance; Oscillations; Solitary wave; Wave nonlinearity; Numerical simulation; FUNWAVE 2.0 model

\section{Introduction}

Harbor resonance is the phenomenon of trapping and amplifying of wave energy inside a 
semi-enclosed water body, such as a bay or harbor. It may be induced by infragravity waves, wave groups, tsunamis, atmospheric fluctuations and shear flow traveling into bays or harbors (Bellotti et al., 2012; Bowers, 1977; De Jong and Battjes, 2004; Dong et al., 2013; Dong et al., 2010a, b; Fabrikant, 1995; Okihiro and Guza, 1996). By creating unacceptable vessel movements, harbor resonance may interrupt the operation of docks and generate excessive mooring forces that may break mooring lines (López and Iglesias, 2014; Uzaki et al., 2010). Rabinovich (2009) reviewed recent advances in understanding and modeling of harbor oscillations.

Among different generation mechanisms, harbor oscillations induced by tsunamis usually have destructive impact. Tsunamis are triggered by submarine earthquakes, offshore landslides, undersea volcanic eruptions, or other kinds of disturbances, such as onshore landslides falling into water, or meteors falling into the open ocean, etc. As tsunamis approach the coastal area, the wave height increases significantly due to the continuous decrease of the water depth and the focus of the wave energy (Zhao et al., 2012). For example, the Indian Ocean tsunami, which was induced by the Sumatra earthquake on December 26, 2004, propagated for about $2 \mathrm{~h}$ to Colombo harbor (Sri Lanka), triggering extreme oscillations with a maximum wave height of $3.87 \mathrm{~m}$ and a resonant period of about $75 \mathrm{~min}$. It then propagated for about $14 \mathrm{~h}$ to Bunbury harbor (Western Australia), triggering oscillations with a maximum wave height of $1.75 \mathrm{~m}$ (Pattiaratchi and Wijeratne, 2009). In order to reduce the disturbance to normal harbor operation and minimize the possible destructive effect, a further research effort is necessary to improve our current knowledge for this type of wave amplification and thus enhance our predictive capability.

Although research efforts on harbor oscillations began in the early 1950s (Vanoni and Carr, 1950), the majority of past studies have been restricted to stationary oscillations induced by the steady wave climate (Bowers, 1977; Dong et al., 2013; Gao et al., 2016a; Ippen and Goda, 1963; Lee, 1971; Mei and Agnon, 1989; Miles and Munk, 1961; Olsen and Hwang, 1971; Raichlen and Naheer, 1976; Vanoni and Carr, 1950; Wang et al., 2011a; Wang et al., 2015; Wang et al., 2014; Wu and Liu, 1990). The study on transient harbor oscillations induced by transient long waves started relatively late and few researchers focused on this problem. Using theoretical and experimental methods, Lepelletier (1980); Lepelletier and Raichlen (1987) studied transient nonlinear oscillations inside the harbor induced by transient long waves, such as tsunamis. Based on a mild-slope equation model, Bellotti (2007) quantified the time-response of harbor basins to 
long waves under resonance conditions. Using laboratory experiments combined with a Boussinesq model (FUNWAVE 2.0), Dong et al. (2010a) explored the response of a rectangular harbor with constant depth to subaerial landslide-generated impact waves. They found that landslide-generated impact waves usually include components with solitary wave characteristics and also components with dispersive wave characteristics. Moreover, the components with solitary characteristics play an important role in the resonance of the harbor. Wang et al. (2011b) developed a second-order dispersive Boussinesq model, which can describe the generation and propagation of earthquake- and landslide-induced tsunamis. Then transient oscillations induced by seafloor movements inside a harbor of constant slope were studied systematically. More recently, using the FUNWAVE 2.0 model, Gao et al. (2016b) carried out a series of numerical experiments on transient harbor oscillations triggered by solitary waves, and further analyzed the relative wave energy distribution systematically inside the harbor by employing the normal mode decomposition method (Gao et al., 2015; Sobey, 2006).

As some characteristics observed in the tsunami events can be well modeled by solitary waves, for instance, the stable form of hump-like waves after a long period of propagation, solitary waves have been employed in tsunami research for decades (e.g., Goring (1978), Synolakis (1987), Liu et al. (1995) and Li (2000)). Studies on solitary waves provide a great deal of information in the investigation of tsunamis, including the runup of solitary waves on a uniform sloping beach (Synolakis, 1987), the interaction of solitary waves with a harbor (Dong et al., 2010a; Gao et al., 2016b), etc. In order to further improve the knowledge of tsunami-induced oscillations, this paper uses solitary waves to explore the related resonant phenomena. The focus of this paper is to comprehensively investigate the wave height evolution, the oscillation amplification, the total wave energy and the relative wave energy distribution during transient harbor oscillations induced by normal-incident solitary waves with different heights. Effects of different bottom profiles inside the harbor on these phenomena are also studied systematically. Compared to Dong et al. (2010a) and Gao et al. (2016b) that have studied transient harbor oscillations excited by solitary waves, there are mainly three research developments in this paper. Firstly, in the above two papers, the incident solitary wave height was relatively small and the wave climate inside the harbor was restricted to weakly nonlinear wave conditions; while in this paper, the wave condition inside the harbor is extended to strong wave nonlinearity close to wave 
breaking. Secondly, investigations on the wave height evolution and the total wave energy inside the harbor are considered for the first time in the present study. Thirdly, in the above two papers, only simple bathymetries inside the harbor, that is, flat bottom and constant slope bottom, were used; whereas in this study, more complex bathymetries inside the harbor are considered. In this paper, identical to Dong et al. (2010a) and Gao et al. (2016b), all numerical experiments are performed using the FUNWAVE 2.0 model as well. For simplification, the harbor is assumed to be long and narrow; the free surface movement inside the harbor then essentially becomes one-dimensional.

The remainder of the paper is organized as follows: Section 2 describes the numerical model, which will be verified using physical experimental data and an analytical solution. Section 3 presents the numerical experiment setup and the experimental wave parameters. Section 4 demonstrates the simulation results, which are explained in detail. Concluding remarks based on the results are given in Section 5.

\section{Numerical model}

\subsection{Model description}

All numerical experiments in this paper are performed using the well-known and widely implemented FUNWAVE 2.0 model. It was proposed and developed at the University of Delaware (Kirby et al., 2003). The one-way wavemaker theory proposed by Chawla and Kirby (2000) is used to generate monochromatic or random waves, while solitary waves are generated by a two-way wavemaker method. Sponge layers are placed at the boundaries of the domain to effectively absorb the energy of outgoing waves with various frequencies and directions. The capability of the FUNWAVE 2.0 model to predict wave propagation and transformation from deep to shallow water has been well validated by laboratory experiments (Bruno et al., 2009; Kirby et al., 2003).

\subsection{Model verification}

To verify the applicability of the FUNWAVE 2.0 model to simulate harbor resonance with strong wave nonlinearity inside the harbor, the model is used to simulate nonlinear harbor resonance experiments of Rogers and Mei (1978). Meanwhile, because the runup of solitary waves on a vertical wall is involved in accurate prediction of solitary wave amplification inside the harbor, the capacity of the model to predict the maximum runup of solitary waves on the 
vertical wall is also examined here. Numerical results are compared with existing experimental data and a second-order analytical solution.

\subsubsection{Nonlinear harbor resonance}

When the incident wave amplitude is small compared to the water depth, oscillations inside the bay can be predicted by linear theory; whereas when the incident amplitude is large, higher harmonic wave components can be expected due to the nonlinear effect. Rogers and Mei (1978) (hereinafter referred to as RM), performed laboratory experiments in a $7.6 \mathrm{~m}$ wide and $13.7 \mathrm{~m}$ long wave tank to study nonlinear oscillations for three bays with lengths of $0.37 \mathrm{~m}, 1.27 \mathrm{~m}$, and $2.18 \mathrm{~m}$ (hereinafter referred to as Bay 1, Bay 2, and Bay 3) and the uniform width of $0.10 \mathrm{~m}$. The wavemaker generated regular waves with a wave period of $1.545 \mathrm{~s}$, and a constant water depth of $0.15 \mathrm{~m}$ was used. In Rogers and Mei (1978), due to nonlinearity, the lengths of the three bays were experimentally determined in accordance with the lowest three resonant modes. According to the linear dispersion relationship

$$
\frac{2 \pi}{T^{2}}=\frac{g}{L} \tanh \left(\frac{2 \pi}{L} h\right),
$$

the wavelength of the regular waves is equal to $1.79 \mathrm{~m}$. The symbols $h, T, L$ and $g$ denote the water depth, the wave period, the wavelength and the gravitational acceleration, respectively.

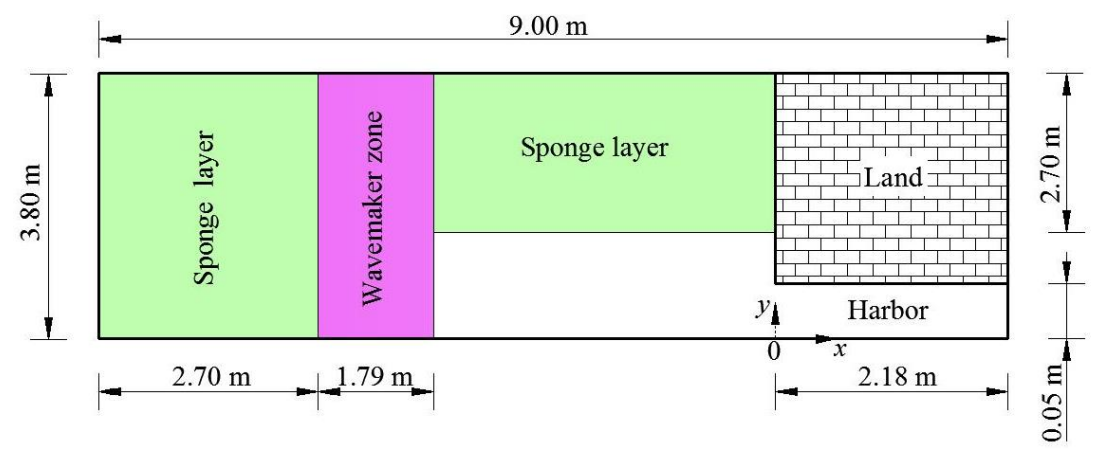

Fig. 1. Schematic plan view of the numerical wave flume used to simulate the experiment of Rogers and Mei (1978) for Bay 3. Since the geometry is symmetric with respect to the $x$-axis, only a half domain is used for computations. The half harbor domain is located in the region $0 \leq x \leq$ $2.18 \mathrm{~m}$ and $0 \leq y \leq 0.05 \mathrm{~m}$.

In this paper, the nonlinear harbor oscillations inside Bays 2 and 3 are simulated. Fig. 1 shows the computational domain of the numerical wave flume used for Bay 3 in the simulation. 
Because the geometry is symmetric with respect to the central line of the bay, only half of the domain is used for simulation.The numerical wave flume has a length of $9.00 \mathrm{~m}$ and a width of $3.80 \mathrm{~m}$. All boundaries are set to be fully reflective. At the left and upper boundaries of the numerical wave flume, sponge layers are installed to absorb the energy of reflected and radiated waves, and the width of the sponge layers is set to be slightly larger than 1.5 times the wavelength of incident regular waves. The target regular wave generated in the internal wavemaker is chosen in such a way that the harmonic amplitudes measured at front of the bay with the entrance closed are the same as RM's measurements. The target wave with wave height $H=0.655 \mathrm{~cm}$, corresponding to the highest incident wave height in RM's experiments, is found to be sufficient to reproduce the RM's mearurements. In the $x$-direction, the grid size $\Delta x$ increases gradually from $0.01 \mathrm{~m}$ inside the harbor to $0.05 \mathrm{~m}$ in the zones of the wavemaker and the sponge layer at the left boundary. Similarly, in the $y$-direction, the grid size $\Delta y$ also increases gradually from $0.01 \mathrm{~m}$ inside the harbor to $0.05 \mathrm{~m}$ outside the harbor. The computational domain consists of 114,742 nodes and 113,980 rectangular elements. A series of wave gauges are deployed equidistantly along the central line of the bay. To obtain a good convergence rate, the Courant number $C_{\mathrm{r}}=\sqrt{g h} \Delta t / \min (\Delta x$, $\Delta y$ ) should be less than 0.5 . Therefore, we use a time step of $\Delta t=0.004 \mathrm{~s}$ in the simulations. The total simulation time is $80.0 \mathrm{~s}$.
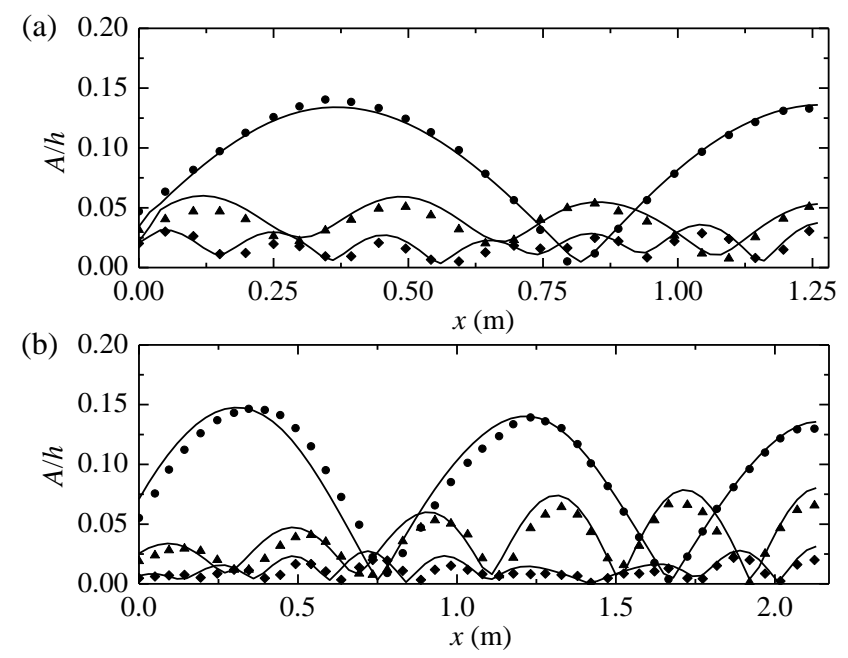

Fig. 2. Comparison of present numerical results with experimental data of Rogers and Mei (1978) for (a) Bay 2 and (b) Bay 3. •, fundamental harmonic; $\boldsymbol{\Lambda}$, second harmonic; $\bullet$, third harmonic; -, present numerical results. 
Fig. 2 compares the numerical results of the first three super harmonics with RM's experimental data for Bays 2 and 3. It should be noted that during each run, data are recorded simultaneously from all wave gauges. After discarding the first $40.0 \mathrm{~s}$ of the time series of the free surface to eliminate the effect of the initial response phase, the remaining $40.0 \mathrm{~s}$ free surface signals are analyzed by using the discrete Fourier transform to obtain amplitudes of the first three super harmonics along the two bays. In Fig. 2, overall agreements are observed between the measured data and the numerical results for all the three super harmonics. For Bay 3 (Fig. 2b), there is a minor phase difference between the numerical and the experimental results at the region $x<1.25 \mathrm{~m}$. It may be caused by the strong wave nonlinearity inside the harbor.

\subsubsection{Solitary wave runup on a vertical wall}

The solitary wave interaction with a fully reflective vertical wall is a classical problem. The maximum runup, $A_{\mathrm{m}}$, on the vertical wall was investigated by Camfield and Street (1969) experimentally. $A_{\mathrm{m}}$ is defined as the vertical elevation above the still water level reached by the water on the vertical wall. Subsequently, Byatt-Smith (1971) deduced a second-order analytical solution by the method of nonlinear superposition of oppositely travelling waves. In this example, the still water depth $h$ is set to $1.0 \mathrm{~m}$. Simulations are implemented for solitary waves with different heights. The length of the computational domain is set to $100.0 \mathrm{~m}$ to ensure that waves of all heights propagate freely over a sufficient distance before hitting the vertical wall. The central line of the wavemaker zone is located in the middle of the domain. Two wave gauges are arranged inside the computational domain; one is placed near the wavemaker zone for recording the incident solitary wave and the other is at the vertical wall for measuring the maximum runup. The simulations are terminated after the solitary waves reflect from the vertical wall. The uniform grid size is $0.1 \mathrm{~m}$. A time step of $0.01 \mathrm{~s}$ is used for these simulations.

The analytical expression of the incident solitary wave can be written as follows (Mei, 1983):

$$
\eta(x, t)=A_{0} \cdot \operatorname{sech}^{2}\left[\sqrt{\frac{3 A_{0}}{4 h^{3}}} \cdot(x-c t)\right], c=\sqrt{g\left(h+A_{0}\right)},
$$

where $A_{0}, x$ and $t$ denote the incident solitary wave height, the $x$-coordinate of a certain position and time, respectively. Fig. 3 illustrates the comparison of the analytical and the simulated incident solitary waves with $A_{0}=0.01 \mathrm{~m}$. It is seen that the solitary wave generated by the 
FUNWAVE 2.0 model agrees well with the analytical expression.

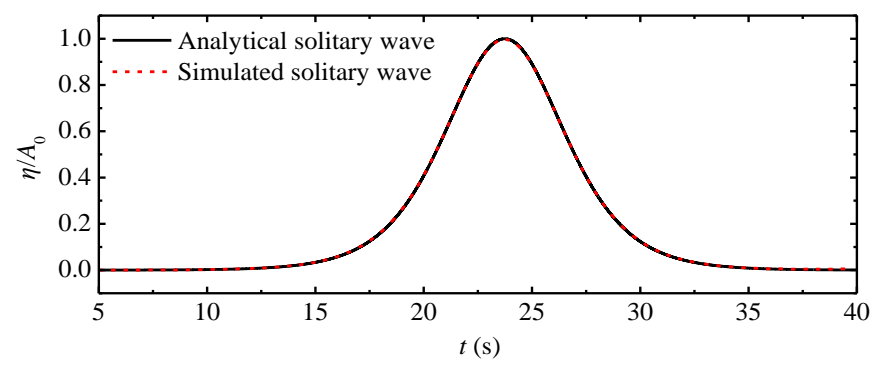

Fig. 3. Comparison of the analytical and the simulated incident solitary waves with $A_{0}=0.01 \mathrm{~m}$

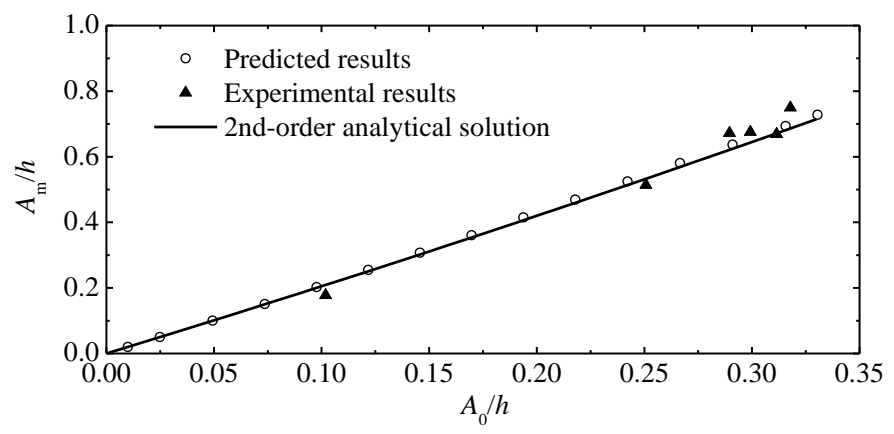

Fig. 4. Verification of the maximum runup of solitary waves on a vertical wall

Fig. 4 shows a comparison of the predicted maximum runup with the experimental data of Camfield and Street (1969) and the second-order analytical solution of Byatt-Smith (1971) which is expressed as

$$
\frac{A_{\mathrm{m}}}{h}=2 \frac{A_{0}}{h}+\frac{1}{2}\left(\frac{A_{0}}{h}\right)^{2}
$$

In general, in the range of the wave nonlinearity considered in these simulations (that is, $\left.A_{\mathrm{m}} / h<0.75\right)$, the present numerical results agree well with the experimental data and the analytical solution.

\section{Numerical setup of the experiment}


(a)

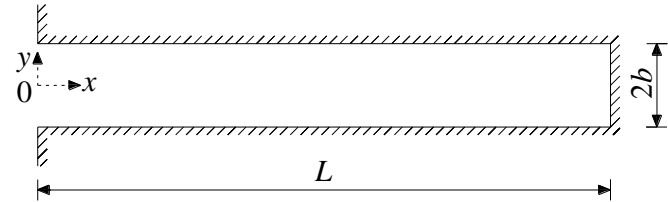

(b)

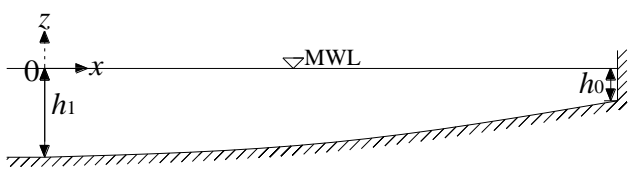

(c)

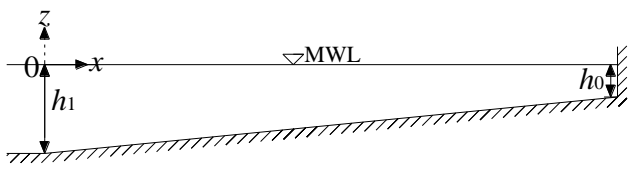

(d)

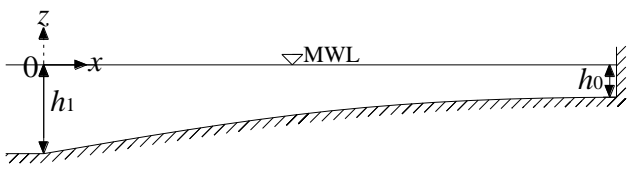

Fig. 5. Definition sketch of elongated rectangular harbors with different bottom profiles: (a) top view of the harbor, (b)-(d) elevation views of the harbors with arc-tangent-type bottom, constant slope bottom and hyperbolic-cosine-type bottom, respectively.

Fig. 5 illustrates the elongated rectangular harbors with different bottom profiles used in the numerical experiments of this paper. In all the numerical experiments the harbors have a length of $L=1000.0 \mathrm{~m}$ and a width of $2 b=20.0 \mathrm{~m}$ (Fig. 5a). Three types of bottom profiles inside the harbors are considered, that is, an arc-tangent-type bottom, a constant slope bottom and a hyperbolic-cosine-type bottom (Fig. 5b-c). Using a horizontal seafloor for the open ocean, the water depth is thus given by

$$
h(x)=\left\{\begin{array}{ll}
h^{\mathrm{I}}(x) & 0 \leq x \leq L,|y| \leq b \\
h_{1} & x<0
\end{array} .\right.
$$

The water depth inside the harbor, $h^{\mathrm{I}}(x)$, can be expressed as

$$
h^{\mathrm{I}}(x)=\left\{\begin{array}{ll}
h_{0}+\alpha \operatorname{atan}[\beta(L-x)] & \text { arc-tangent-type bottom } \\
h_{1}-\gamma x & \text { constant slope bottom } \\
h_{0} \cosh ^{\kappa}[\mu(L-x)] & \text { hyperbolic-cosine-type bottom }
\end{array},\right.
$$

where $h_{0}$ denotes the local water depth at the backwall. The water depth outside the harbor, $h_{1}$, in all the numerical experiments is set to $14.0 \mathrm{~m}$. The symbols $\alpha, \beta, \gamma, \kappa$ and $\mu$ are geometrical parameters determining bottom profiles inside the harbors, which satisfy the following relationships: 


$$
\begin{gathered}
\beta=\frac{1}{L} \tan \left(\frac{h_{1}-h_{0}}{\alpha}\right), \\
\gamma=\frac{h_{1}-h_{0}}{L},
\end{gathered}
$$

and

$$
\mu=\frac{1}{L} \operatorname{acosh}\left[\left(\frac{h_{1}}{h_{0}}\right)^{1 / \kappa}\right]
$$

It should be noticed here that although the numerical model FUNWAVE 2.0 is two-dimensional, the topographic changes inside the harbors used in numerical experiments are one-dimensional (i.e. occurring only in the $x$-direction).

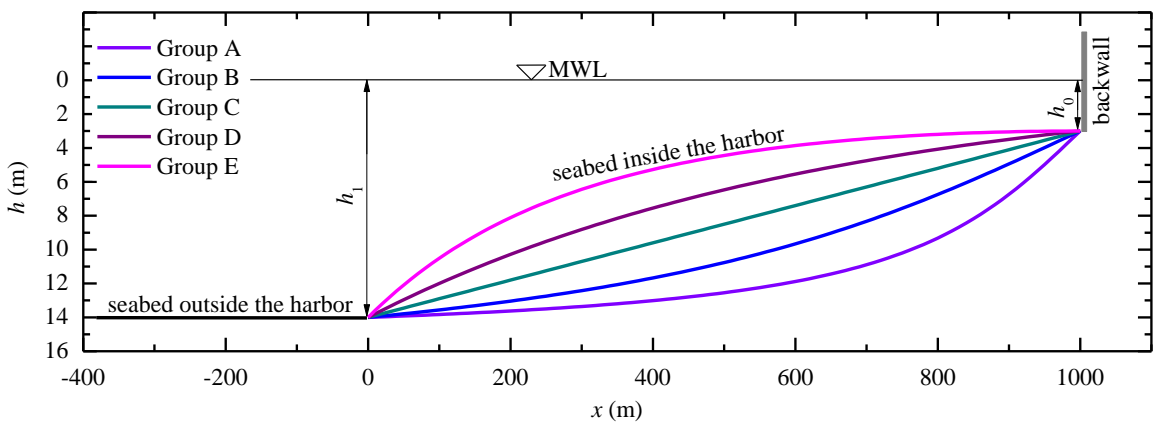

Fig. 6. Topographical variations inside the harbors used in Groups A-E

Table 1 Geometric parameters of the harbors and wave parameters of the incident solitary waves used in all the numerical experiments

\begin{tabular}{cccc}
\hline Group & Topography & Geometrical parameters & $A_{0}$ \\
\hline A & \multirow{2}{*}{ arc-tangent-type } & $h_{1}=14 \mathrm{~m}, h_{0}=3 \mathrm{~m}, \alpha=8 \mathrm{~m}, \bar{h}=11.40 \mathrm{~m}$ & $0.02 \mathrm{~m}-0.52 \mathrm{~m}$ \\
\cline { 3 - 4 } & & $h_{1}=14 \mathrm{~m}, h_{0}=3 \mathrm{~m}, \alpha=10 \mathrm{~m}, \bar{h}=9.98 \mathrm{~m}$ & $0.02 \mathrm{~m}-0.48 \mathrm{~m}$ \\
\hline B & constant slope & $h_{1}=14 \mathrm{~m}, h_{0}=3 \mathrm{~m}, \bar{h}=8.50 \mathrm{~m}$ & $0.02 \mathrm{~m}-0.42 \mathrm{~m}$ \\
\hline C & \multirow{2}{*}{ hyperbolic-cosine-type } & $h_{1}=14 \mathrm{~m}, h_{0}=3 \mathrm{~m}, \kappa=0.005, \bar{h}=7.13 \mathrm{~m}$ & $0.02 \mathrm{~m}-0.35 \mathrm{~m}$ \\
\cline { 3 - 4 } & & $h_{1}=14 \mathrm{~m}, h_{0}=3 \mathrm{~m}, \kappa=20.0, \bar{h}=5.65 \mathrm{~m}$ & $0.02 \mathrm{~m}-0.30 \mathrm{~m}$ \\
\hline E & &
\end{tabular}

Table 1 presents the geometric parameters of the harbors and the wave parameters of the incident solitary waves that have been used. The mean water depth inside the harbor, $\bar{h}$, can be calculated using the following formula:

$$
\bar{h}=\frac{1}{L} \int_{0}^{L} h^{\mathrm{I}}(x) d x .
$$

Five groups of numerical experiments are considered, namely Groups A-E. All the harbors used in 
these groups have the same water depth at the harbor entrance, $h_{1}=14.0 \mathrm{~m}$, and at the backwall, $h_{0}=3.0 \mathrm{~m}$, but have different bottom profiles. Fig. 6 illustrates the topographical variations inside the harbors used in these groups. In Groups A and B, both the harbors have an arc-tangent-type bottom. However, due to the variation of $\alpha$, the mean water depths inside the two harbors are different, which are $11.40 \mathrm{~m}$ and $9.98 \mathrm{~m}$, respectively. Similarly, although both the harbors in Groups D and E have a hyperbolic-cosine-type bottom, the mean water depths, which are $7.13 \mathrm{~m}$ and $5.65 \mathrm{~m}$, respectively, are different due to different values of $\kappa$. In Group C, the harbor has a constant slope bottom, and the mean water depth is $8.5 \mathrm{~m}$, which falls between those of Groups B and D. In each group, the incident solitary wave height from the open ocean, $A_{0}$, starts from 0.02 $\mathrm{m}$ and increases gradually to a maximum value. The maximum height of the incident solitary wave is determined according to the maximum wave nonlinearity parameter inside the harbor, $A_{\mathrm{m}} / h_{0} . A_{\mathrm{m}}$ denotes the maximum oscillation of the solitary wave inside the harbor, which can be obtained through the simulation. In each group, the maximum value of $A_{\mathrm{m}} / h_{0}$ is set to be slightly larger than 0.7 (this will be described in detail in Section 4), and less than 0.78 which is a critical value for the breaking of the solitary wave (McCowan, 1894). In fact, in each group, some additional cases with larger incident solitary waves have also been simulated. However, they are not considered in this paper because wave breaking occurs inside the harbor.

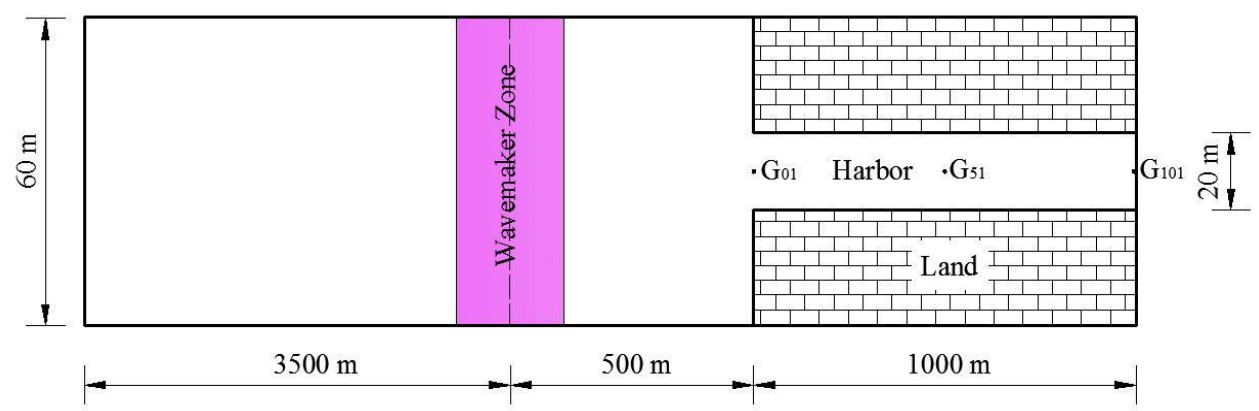

Fig. 7. Schematic plan view of the numerical wave tank used in the numerical experiments. The definition of the coordinate system is identical to that of Fig. 5.

Fig. 7 demonstrates the numerical wave tank used in the numerical experiments. There are 101 wave gauges $\left(\mathrm{G}_{01}-\mathrm{G}_{101}\right)$ deployed equidistantly with a spacing $10.0 \mathrm{~m}$ along the center line of the harbor. Gauges $G_{01}$ and $G_{101}$ are those at the entrance and the backwall of the harbor, 
respectively. Dimensions of the numerical wave tank are $5000.0 \mathrm{~m} \times 60.0 \mathrm{~m}$. All the boundaries are set to be fully reflective. A two-way wavemaker is installed $500.0 \mathrm{~m}$ from the harbor entrance. Due to the poor energy dissipation of numerical solitary waves, the sponge layer has not been established at the left boundary. To ensure that the backward propagating component of the generated waves is not reflected to the harbor during our simulations, the distance between the left wall of the flume and the central line of the wavemaker zone is extended to $3500.0 \mathrm{~m}$. The grid sizes $\Delta x$ and $\Delta y$ are uniform, $1.0 \mathrm{~m}$ over the whole computational domain. Total numbers of the grid nodes and the rectangular elements are 305,061 and 300,000, respectively. The simulation time is $600.0 \mathrm{~s}$ with a time step of $0.05 \mathrm{~s}$.

\section{Results and discussion}

4.1 Time series of the wave elevation

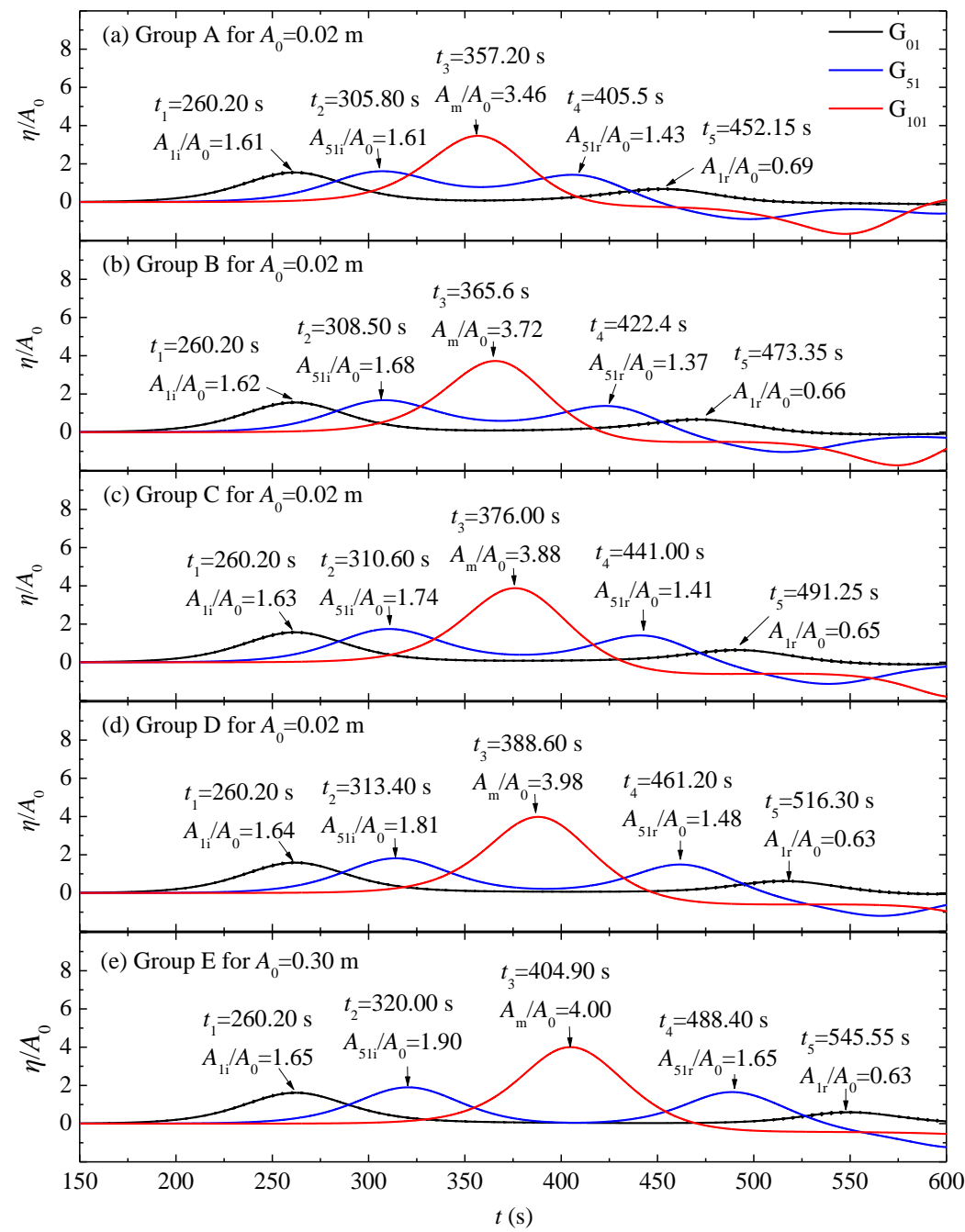

Fig. 8. Time series of the wave elevations at gauges $\mathrm{G}_{01}, \mathrm{G}_{51}$ and $\mathrm{G}_{101}$ for $A_{0}=0.02 \mathrm{~m}$ in Groups A-E 
The time series of the wave elevations at gauges $\mathrm{G}_{01}, \mathrm{G}_{51}$ and $\mathrm{G}_{101}$ for the incident solitary wave height, $A_{0}=0.02 \mathrm{~m}$, in Groups A-E are shown in Fig. 8 . It is noted that in this figure, the time series of the wave elevations are normalized by $A_{0} . A_{n \mathrm{i}}$ and $A_{n \mathrm{r}}(n=1,51)$ denote the heights of the incident wave and the reflected wave from the backwall at the $n$-th gauge, respectively. Due to the same height of the incident solitary wave and the same water depth outside the harbor, all the wave elevations at gauge $\mathrm{G}_{01}$ reach a maximum at $t_{1}=260.20 \mathrm{~s}$. As the incident wave further propagates inside the harbor, differences of the wave elevations in these groups emerge. Because the mean water depth decreases gradually from Group A to E, the mean wave propagation velocity inside these harbors decreases correspondingly. Therefore, the values of $t_{2}-t_{5}$ increase from Group A to $\mathrm{E}$. Take the moment, $t_{3}$, in which the incident wave reaches the maximum oscillation on the backwall, as an example. $t_{3}$ increases from $357.20 \mathrm{~s}$ in Group A to $404.90 \mathrm{~s}$ in Group E, with an increment of $47.7 \mathrm{~s}$. Similarly, for the same reason, the amplification factor of the incident solitary wave, $A_{\mathrm{m}} / A_{0}$, increases gradually from 3.46 in Group A to 4.00 in Group E. The time series of the wave elevations at gauges $G_{01}$ and $G_{51}$ show that the height of the reflected wave is obviously smaller than that of the incident wave inside the harbor.

To illustrate the influence of different heights of the incident solitary waves on the wave climate inside the harbor, the time series of the wave elevations at gauges $G_{01}, G_{51}$ and $G_{101}$ corresponding to the incident solitary wave with the maximum height in Groups A-E are illustrated in Fig. 9. The first thing to point out is that the maximum nonlinearity parameter inside the harbor, $A_{\mathrm{m}} / h_{0}$, in Groups A-E is $0.70,0.72,0.74,0.70$ and 0.71 , respectively. It can be seen that the time series of the wave elevations at these gauges show significant nonlinear deformation. The nonlinear deformation is reflected concretely in the following two aspects. Firstly, obvious asymmetry can be observed in the time series of wave elevations in all the five cases. For example, at gauge $\mathrm{G}_{101}$, the free surface promptly rises from zero to the maximum runup $A_{\mathrm{m}}$, while the subsequent process that the free surface falls from $A_{\mathrm{m}}$ to zero takes much more time. Secondly, there exist secondary peaks at the free surface. The free surface of the reflected wave at gauge $\mathrm{G}_{51}$ shows several secondary peaks. When the reflected wave reaches the harbor entrance, it finally evolves into a small leading wave with solitary wave characteristics followed by a dispersive wave tail. Fig. 9 also shows that the height of the reflected wave is always larger than that of the incident wave at gauge $\mathrm{G}_{51}$, which is contrary to the observation shown in Fig. 8. To demonstrate 
this phenomenon more evidently, Fig. 10 shows the variation of the dimensionless heights of the incident and the reflected waves at gauge $\mathrm{G}_{51}$ with respect to the incident solitary wave height, $A_{0}$, in all groups. The dimensionless height of the incident wave, $A_{51 \mathrm{i}} / A_{0}$, seems not sensitive to the change of $A_{0}$. However, the dimensionless height of the reflected wave, $A_{51 \mathrm{r}} / A_{0}$, shows an obvious gradual increase with an increase of $A_{0}$. When $A_{0}$ is small, the value of $A_{511} / A_{0}$ is always larger than that of $A_{51 \mathrm{r}} / A_{0}$. However, with an increase of $A_{0}$, the latter gradually approaches and exceeds the former. The less the mean water depth is, the more obvious the tendency becomes. The changing curves of $\left(A_{51 \mathrm{r}}-A_{51 \mathrm{i}}\right) / A_{0}$ are almost parallel to those of $A_{51 \mathrm{r}} / A_{0}$ in all groups.

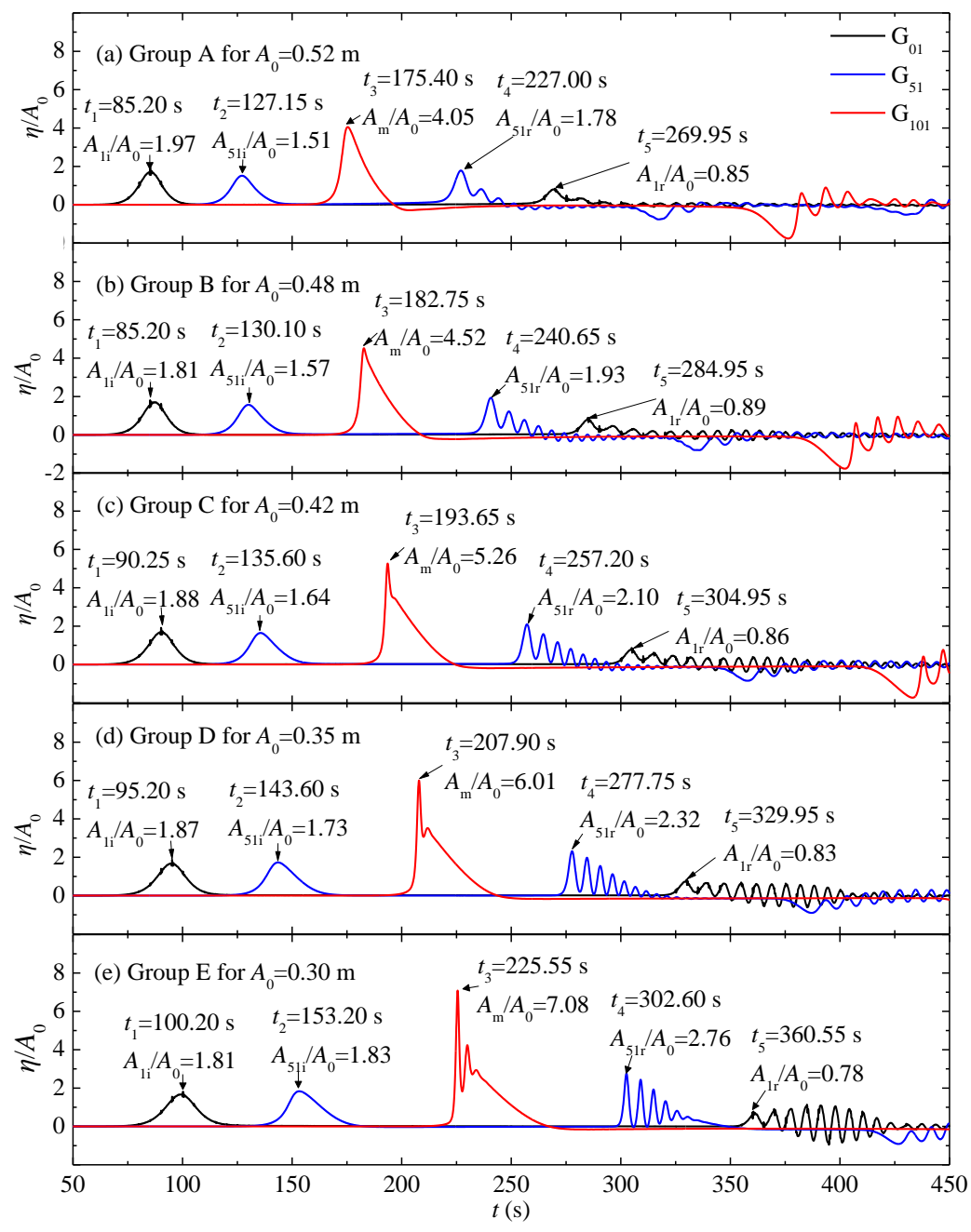

Fig. 9. Time series of the wave elevations at gauges $\mathrm{G}_{01}, \mathrm{G}_{51}$ and $\mathrm{G}_{101}$ corresponding to the incident solitary wave with the maximum height in Groups A-E 

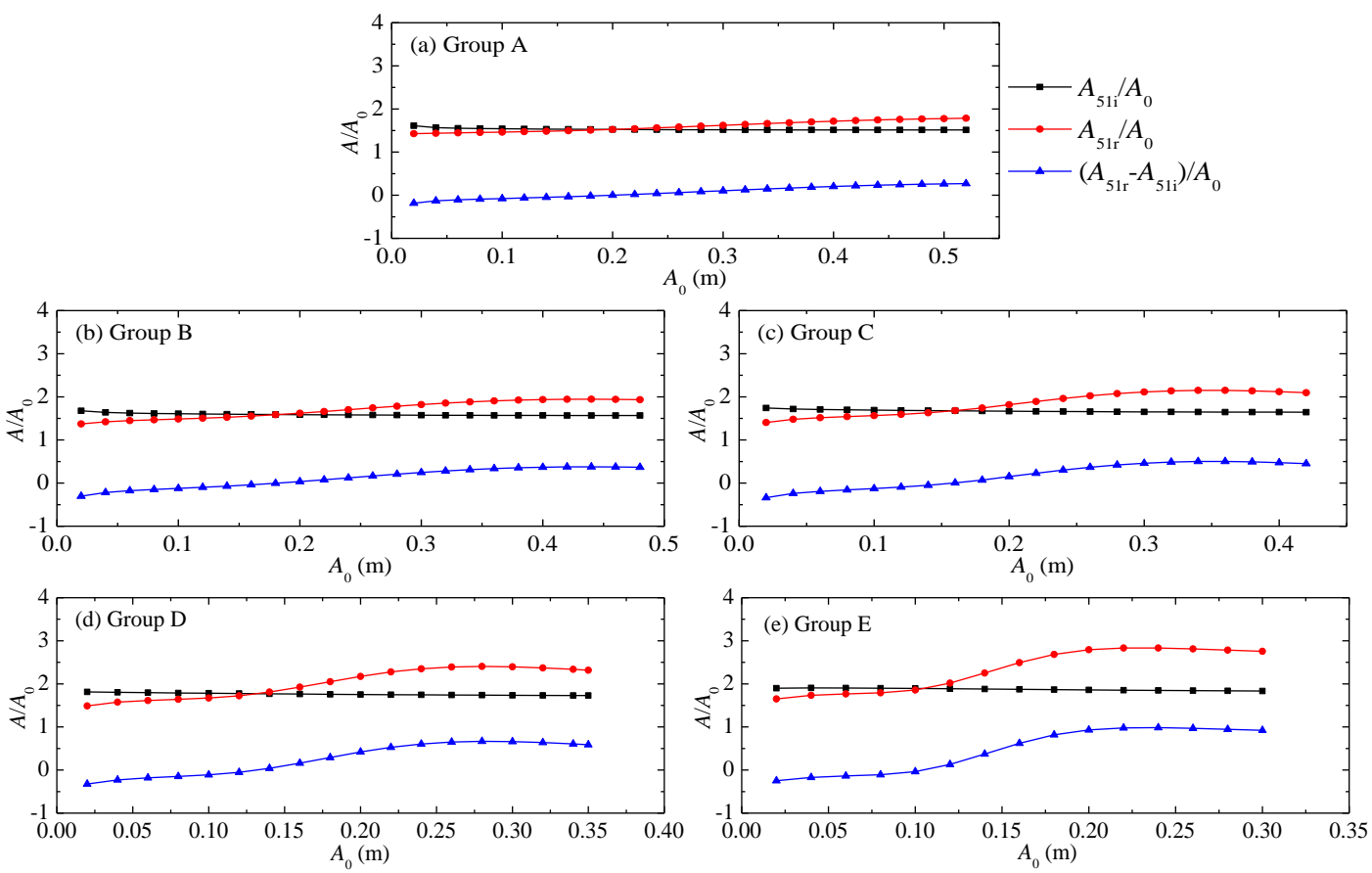

Fig. 10. Changing curves of the dimensionless heights of the incident and the reflected waves at gauge $\mathrm{G}_{51}$ with respect to the height of the incident solitary wave from the open sea (the upper two curves). The difference of the two curves is shown by the lower curve.

In Figs. 9 and 10, the height of the reflected wave becomes larger than that of the incident wave at gauge $\mathrm{G}_{51}$ when the incident solitary wave is large. To qualitatively explain this phenomenon, the incident and the reflected waveforms at time $t_{2}$ and $t_{4}$ in the cases with the largest incident solitary wave in Group A-E are illustrated in Fig. 11. $t_{2}$ denotes the moment at which the wave crest of the incident wave reaches gauge $\mathrm{G}_{51}(x=500.0 \mathrm{~m})$, and $t_{4}$ denotes the moment at which the first wave crest of the reflected wave passes through gauge $\mathrm{G}_{51}$. The values of $t_{2}$ and $t_{4}$ in this figure are identical to those in Fig. 9. It can be clearly seen in Fig. 11 that for the large incident solitary wave, the reflected waveform has a maximum wave crest, which exceeds the peak of the incident waveform, followed by several secondary crests. The local waveform of the reflected wave around the first wave crest has a narrower spatial span, and the local wave energy there becomes more concentrated, which causes the above phenomenon although the total energy of the incident wave is larger than or equal to that of the reflected wave. 


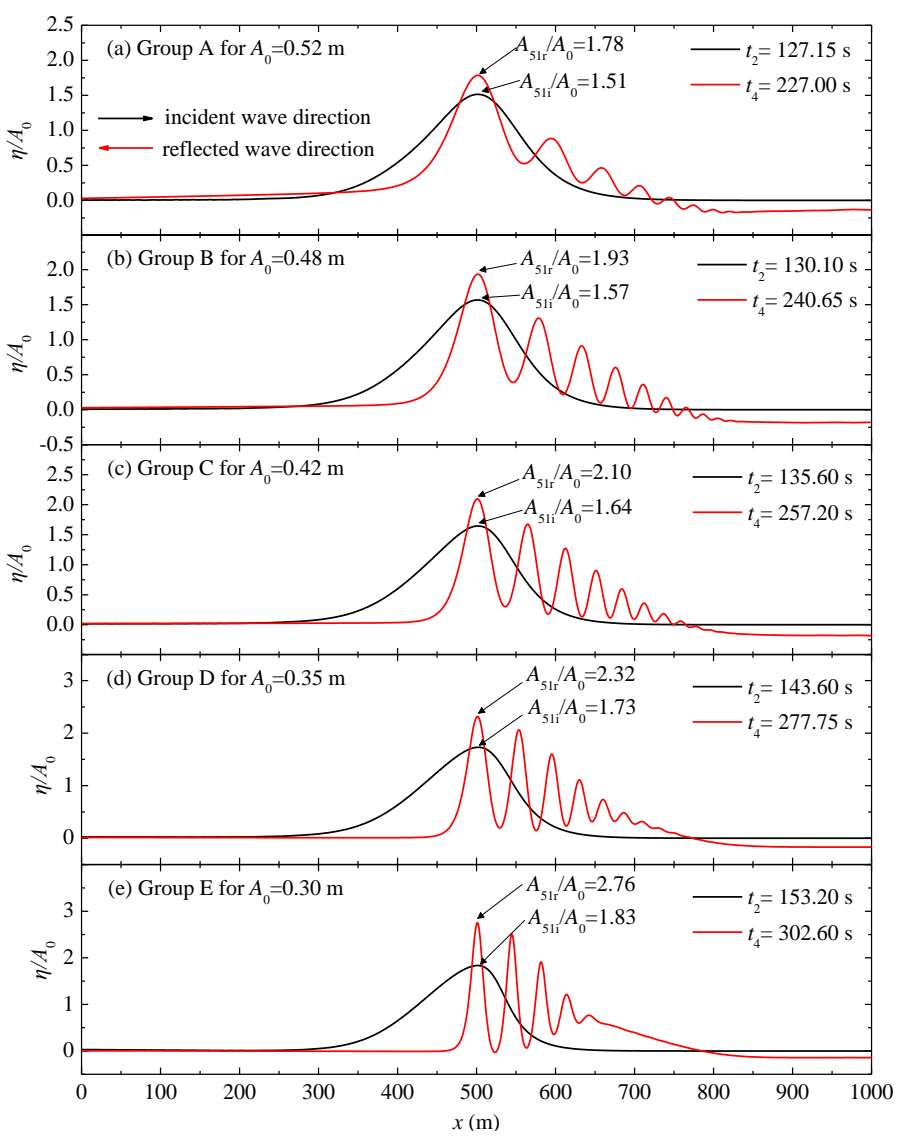

Fig. 11. The incident and the reflected waveforms at time $t_{2}$ and $t_{4}$ in the cases with the largest incident solitary wave in Group A-E

\subsection{Wave height evolution}

When the incident solitary wave propagates over the mild slope inside the harbor, the wave height is expected to increase with the decrease of the water depth. Through the numerical simulation, the wave height evolution of the incident and the reflected solitary waves inside the harbors with different bottom profiles can be obtained directly. Fig. 12 shows the wave height evolution of the incident and the reflected waves inside the harbors for $A_{0}=0.02 \mathrm{~m}$ in all groups. For all the groups, due to the gradual decrease of the water depth as the incident wave propagates to the backwall, the height of the incident wave gradually increases as expected. It is found that the height of the reflected wave is always less than that of the incident wave at $x<800.0 \mathrm{~m}$. According to the linear wave theory and the wave energy conservation principle, if the wave reflection is ignored during the shoaling process, the wave height evolution of the linear long wave propagating over the mild slope can be analytically predicted by the following formula 


$$
\frac{A}{A_{\mathrm{R}}}=\left(\frac{h_{\mathrm{R}}}{h}\right)^{1 / 4},
$$

in which $h_{\mathrm{R}}$ and $A_{\mathrm{R}}$ denote the water depth and the wave height at a certain reference position, respectively. This $1 / 4$ power dependence is often called Green's law (Mei, 1983). The wave height evolution of the incident wave inside the harbor predicted by Eq. (10) is also demonstrated in Fig. 12. To eliminate the entrance boundary effect of gauge $G_{01}$, gauge $G_{02}$ is chosen as the reference position in the analysis. In general, the predictions of Green's law agree well with the numerical results in the whole domain except the area in which the incident and the reflected waves interact with each other near the backwall. For Group E, the analytical prediction is slightly larger than the numerical result. This is due to the fact that in Group E the relatively large wave energy reflection occurs during the shoaling process, which will be elaborated below.

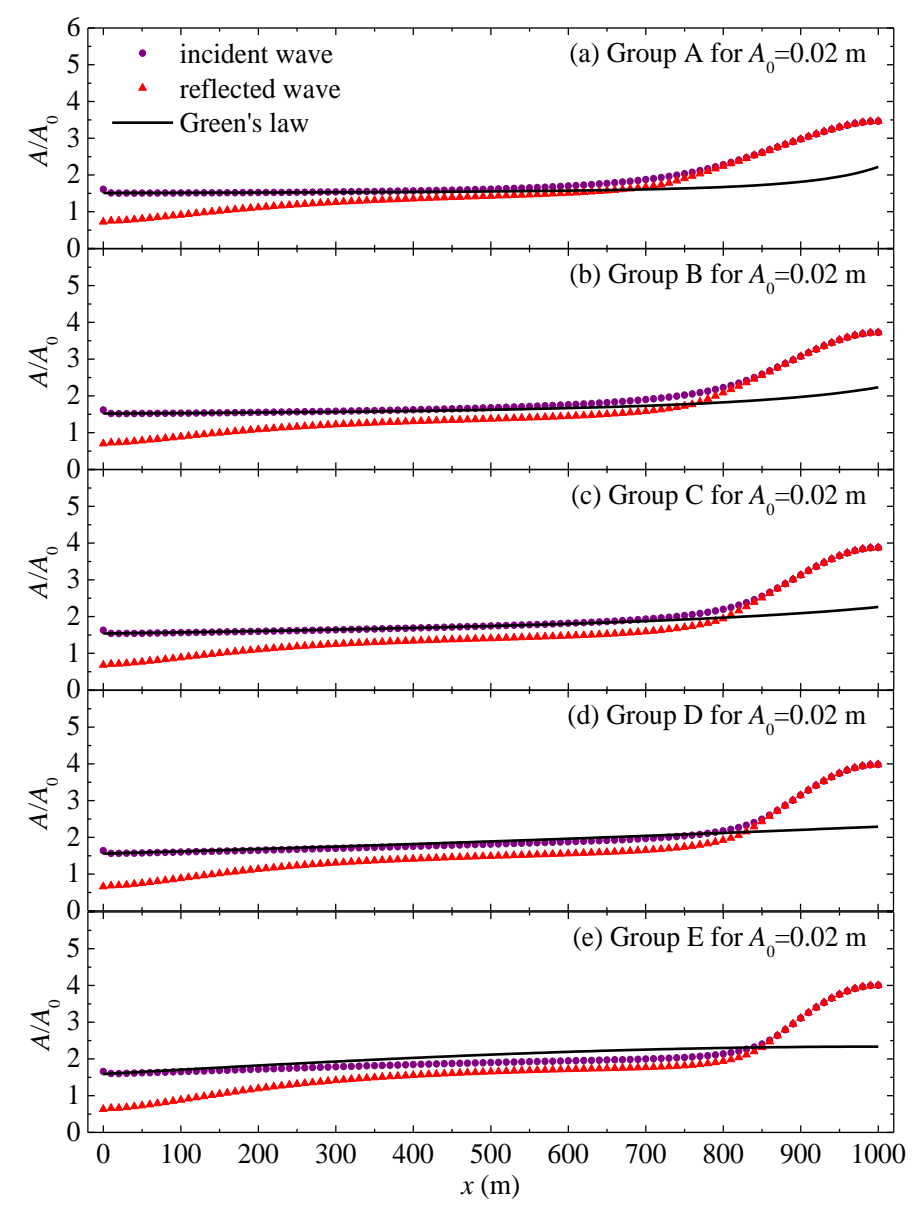

Fig. 12. Wave height evolution of the incident and the reflected waves inside the harbor for $A_{0}=0.02 \mathrm{~m}$ in Groups A-E

Fig. 13 shows the wave height evolution of the incident and the reflected waves, inside the harbors with different bottom profiles, corresponding to the incident solitary wave with the 
maximum height in all groups. When the wave nonlinearity inside the harbor increases, the height evolution of the incident wave still coincides well with Green's law except near the backwall. However, the height of the reflected wave becomes larger than that of the incident wave in almost the whole harbor except near the entrance, which is consistent with the phenomenon shown in Figs. 9-11. From the viewpoint of tsunami-induced inundation, the reflected wave inside the harbor is more likely to cause submersion near the port zone. It should be noted that the reason the height of the reflected wave drops below that of the incident wave near the harbor entrance is that when the reflected wave crest reaches the harbor entrance, part of the reflect wave energy has already been transmitted into the open sea through wave diffraction at the entrance.

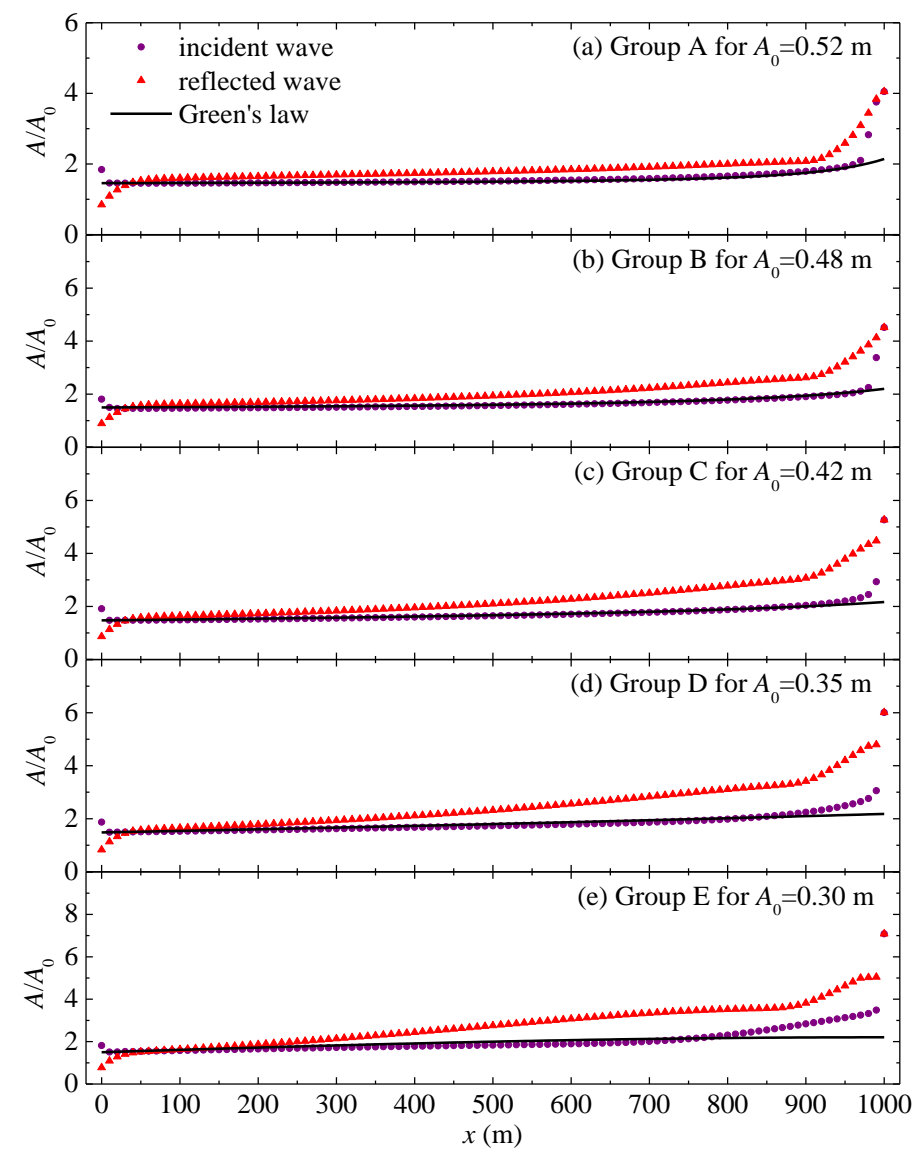

Fig. 13. Wave height evolutions of the incident and the reflected waves inside the harbor corresponding to the incident solitary wave with the maximum height in Groups A-E

\subsection{Solitary wave amplification}

To investigate the relationship between the incident solitary wave height, $A_{0}$, and the maximum oscillation inside the harbor, $A_{\mathrm{m}}$, Dong et al. (2010a) and Gao et al. (2016b) carried out 
a series of numerical simulations and found that the maximum oscillation inside the harbor with flat or constant-slope bottom grows linearly with the incident solitary wave height. However, in their studies, the wave climate inside the harbor was limited to the weakly nonlinear wave condition. In the current study, this discussion is extended to strong wave nonlinearity prior to wave breaking. Fig. 14 shows the changes of the maximum oscillation at gauge $\mathrm{G}_{101}$ with respect to the incident solitary wave height in all groups. It can be seen that when the incident solitary wave height is less than $0.15 \mathrm{~m}$, the maximum oscillation inside the harbor can be regarded as growing linearly with it. However, when the incident solitary wave height increases, the nonlinear effect appears. Moreover, the less the mean water depth inside the harbor is, the more obvious the nonlinear effect is, which illustrates that the bottom profile inside the harbor is one of the vital factors determining the amplification of the transient long waves, especially when the transient long waves are large.

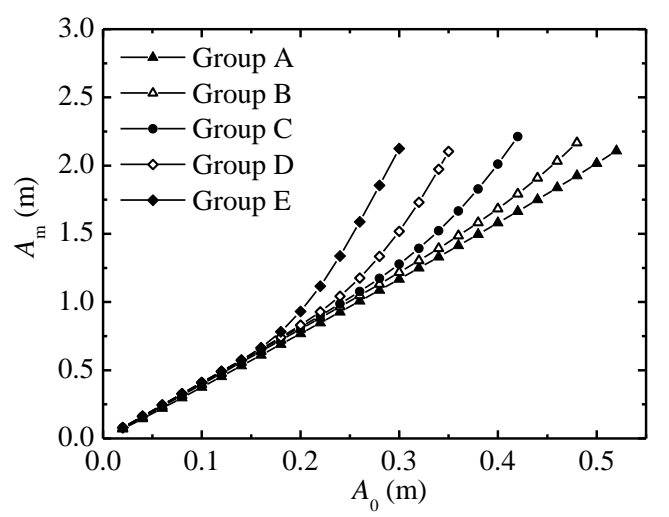

Fig. 14. Maximum oscillations at gauge $\mathrm{G}_{101}$ for all cases in Groups A-E

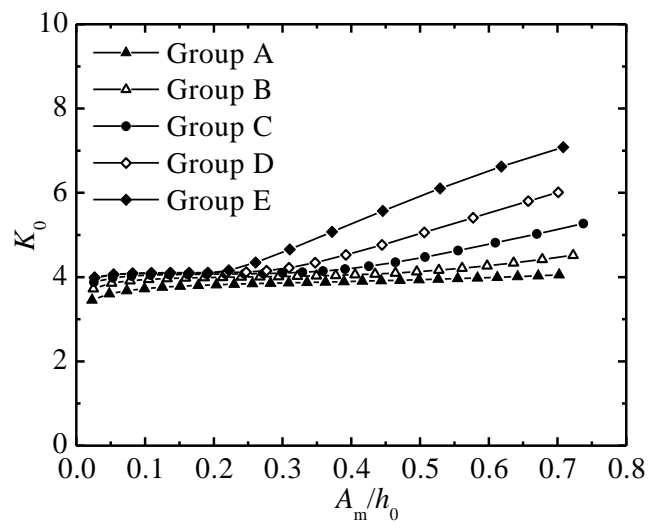

Fig. 15. Amplification factors of incident solitary waves inside the harbor, $K_{0}$, for all cases in Groups A-E 
Dong et al. (2010a) pointed out that the maximum oscillation induced by solitary waves can be estimated by the formula

$$
A_{\mathrm{m}}=K_{0} A_{0},
$$

where the parameter $K_{0}$ was interpreted as the amplification factor of the response of the harbor to a transient long wave. To visually demonstrate the relationship between the amplification factor, the wave nonlinearity parameter and the bottom profile inside the harbor, Fig. 15 shows the amplification factors for all numerical experiments in Groups A-E. It can be seen that for a relatively weak nonlinearity range $\left(A_{\mathrm{m}} / h_{0}<0.2\right)$, as expected, $K_{0}$ is generally not sensitive to the wave nonlinearity parameter, $A_{\mathrm{m}} / h_{0}$, and can be approximately regarded as a constant. As $A_{\mathrm{m}} / h_{0}$ increases further, the value of $K_{0}$ increases gradually with the wave nonlinearity parameter. Moreover, the increasing rate of $K_{0}$ is closely related to the bottom profile. The less the mean water depth is inside the harbor, the larger the increase of the rate of $K_{0}$.
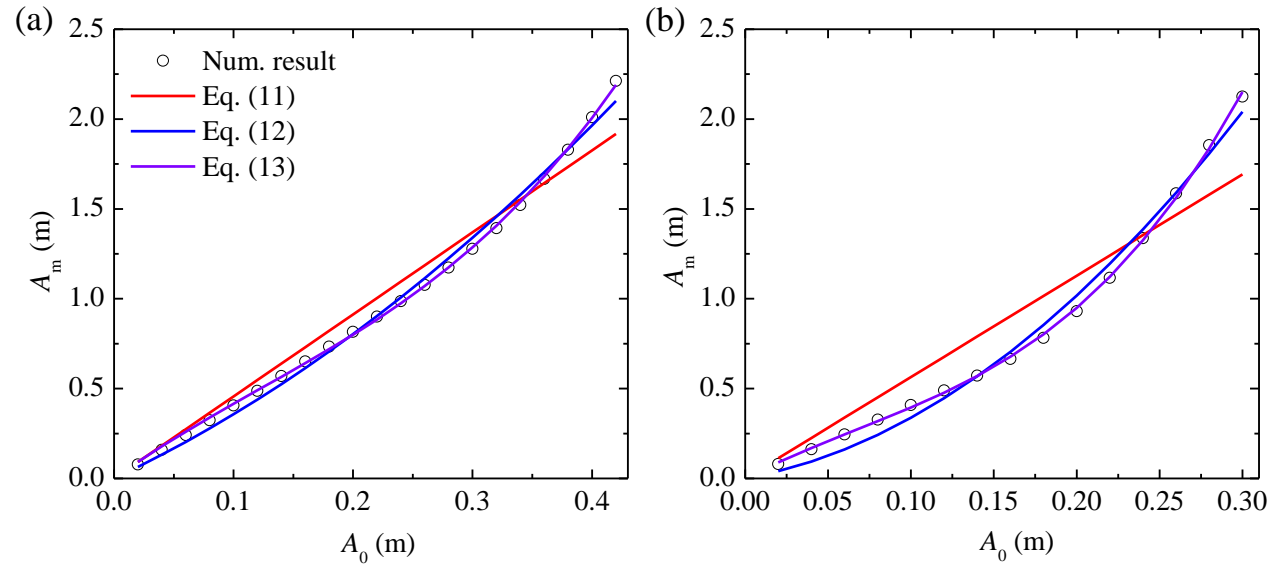

Fig. 16. Maximum oscillations at gauge $\mathrm{G}_{101}$ in the numerical experiments and the fitted curves of Eqs. (11)-(13) using the least squares method for (a) Group C and (b) Group E

In this paper, by adding additional nonlinear correction terms, Eq. (11) proposed by Dong et al. (2010a) is modified to

$$
A_{\mathrm{m}}=K_{1} A_{0}+C_{1} A_{0}^{2},
$$

or

$$
A_{\mathrm{m}}=K_{2} A_{0}+C_{2} A_{0}^{2}+D A_{0}^{3},
$$

in which $K_{1}, K_{2}, C_{1}, C_{2}$ and $D$ are unknown coefficients. By using the least squares method, the 
numerical data shown in Fig. 14 are fitted with Eqs. (11)-(13). Fig. 16 shows the fitted results of the three equations for Groups $\mathrm{C}$ and $\mathrm{E}$. It can be found that in the range of wave nonlinearity studied in this paper, the fitted curves of Eq. (13) show optimal correlation with the numerical data, while those of Eq. (11) show the worst agreement. Table 2 presents the values of the parameters $K_{i}$ ( $i=0,1$ and 2$), C_{j}$ ( $j=1$ and 2), $D$, the correlation coefficient $R^{2}$ and the root mean square errors RMSE in all groups. The quantitative results show that the fitted curves of Eq. (13) in all the groups have the maximum values of $R^{2}$ and the minimal values of $R M S E$, while the fitted curves of Eq. (11) have the minimal values of $R^{2}$ and the maximum values of RMSE. This coincides with the phenomena shown in Fig. 16. Therefore, when strong wave nonlinearity occurs inside the harbor, the maximum oscillation induced by the transient long wave is not well predicted by the linear equation proposed by Dong et al. (2010a), whereas Eq. (13) with the terms of $A_{0}{ }^{2}$ and $A_{0}{ }^{3}$ seems to be able to accurately describe the amplification of the transient long waves inside the harbor.

Table 2 Values of the parameters $K_{i}\left(i=0,1\right.$ and 2), $C_{j}\left(j=1\right.$ and 2), $D$, the correlation coefficient $R^{2}$ and the root mean square errors $R M S E$ in all groups

\begin{tabular}{|c|c|c|c|c|c|c|c|c|c|c|c|c|}
\hline \multirow{2}{*}{ Group } & \multicolumn{3}{|c|}{$A_{\mathrm{m}}=K_{0} A_{0}$} & \multicolumn{4}{|c|}{$A_{\mathrm{m}}=K_{1} A_{0}+C_{1} A_{0}^{2}$} & \multicolumn{5}{|c|}{$A_{\mathrm{m}}=K_{2} A_{0}+C_{2} A_{0}^{2}+D A_{0}^{3}$} \\
\hline & $K_{0}$ & $R^{2}$ & $R M S E$ & $K_{1}$ & $C_{1}$ & $R^{2}$ & $R M S E$ & $K_{2}$ & $C_{2}$ & $D$ & $R^{2}$ & $R M S E$ \\
\hline A & 3.958 & 0.9988 & 0.02164 & 3.694 & 0.6632 & 1.0 & 0.00318 & 3.737 & 0.3786 & 0.3959 & 1.0 & 0.00287 \\
\hline B & 4.216 & 0.9936 & 0.04996 & 3.613 & 1.643 & 0.9989 & 0.02122 & 4.218 & -2.482 & 6.32 & 0.9998 & 0.00815 \\
\hline $\mathrm{C}$ & 4.564 & 0.9707 & 0.1068 & 3.124 & 4.470 & 0.9939 & 0.04878 & 4.752 & -8.177 & 22.09 & 0.9995 & 0.01430 \\
\hline $\mathrm{D}$ & 5.009 & 0.9104 & 0.1701 & 2.373 & 9.528 & 0.9863 & 0.06892 & 5.436 & -17.64 & 54.93 & 0.9997 & 0.01138 \\
\hline $\mathrm{E}$ & 5.642 & 0.8975 & 0.2032 & 1.670 & 17.10 & 0.9895 & 0.06755 & 4.771 & -16.35 & 81.16 & 0.9994 & 0.01667 \\
\hline
\end{tabular}

\subsection{Total wave energy and relative wave energy distribution}

Gao et al. (2016b) systematically analyzed the relative wave energy distribution under the transient harbor oscillation induced by solitary waves through the normal mode decomposition method (hereinafter referred to as the NMD method). The NMD method was proposed originally by Sobey (2006) to calculate the eigenfrequencies, the eigenmodes and the response amplitudes of different modes in natural harbors that are subjected to storm tides or tsunamis. The method was improved by Gao et al. (2015) to predict the eigenfrequencies and eigenmodes more accurately. In 
this section, the NMD method is used to investigate the effect of different bottom profiles on the total wave energy and the relative wave energy distribution inside the harbor. For the theory of the NMD method, the interested reader is referred to Sobey (2006) and Gao et al. (2015).

(a)

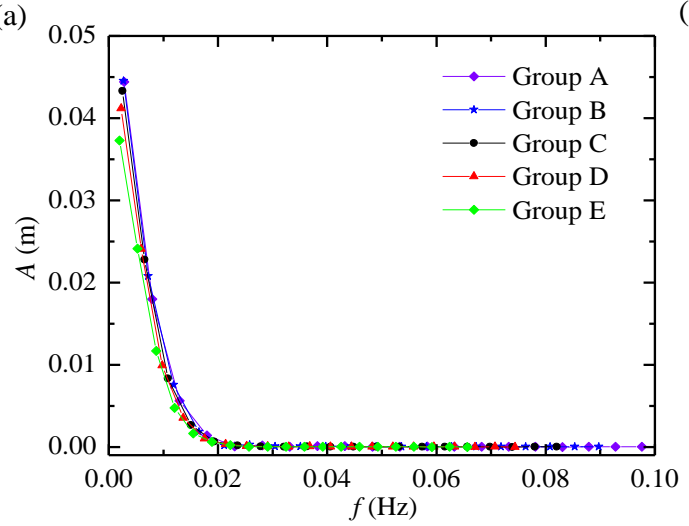

(b)

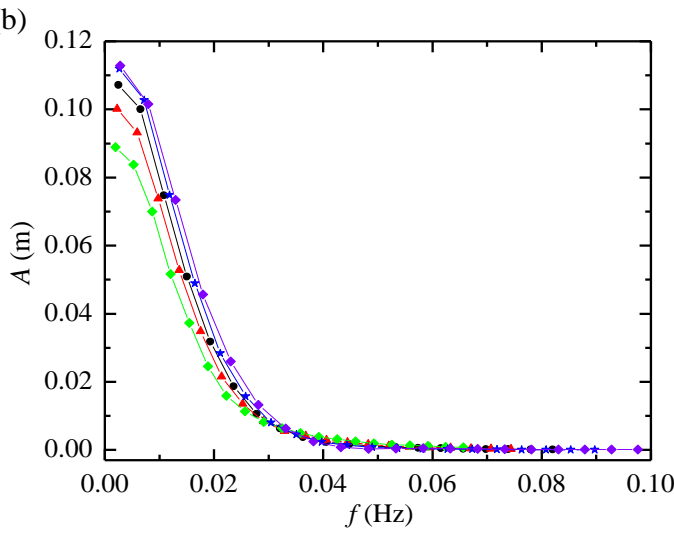

Fig. 17. Response amplitudes of the lowest twenty resonant modes of the harbors used in Groups A-E for (a) $A_{0}=0.02 \mathrm{~m}$ and (b) $A_{0}=0.1 \mathrm{~m}$

Table 3 Numerical fitting errors (NFEs) of the NMD method for the cases shown in Fig. 17

\begin{tabular}{cccccc}
\hline$A_{0}(\mathrm{~m})$ & Group A & Group B & Group C & Group D & Group E \\
\hline 0.02 & $0.029 \%$ & $0.027 \%$ & $0.219 \%$ & $0.025 \%$ & $0.112 \%$ \\
0.10 & $1.303 \%$ & $1.328 \%$ & $1.355 \%$ & $2.073 \%$ & $3.997 \%$ \\
\hline
\end{tabular}

Fig. 17 shows the amplitude distributions of the lowest twenty resonant modes of the harbors used in Groups A-E for $A_{0}=0.02 \mathrm{~m}$ and $A_{0}=0.1 \mathrm{~m}$. To guarantee the accurate prediction of the response amplitudes of different resonant modes, the numerical fitting errors (NFEs) of the NMD method in these cases are all less than $4 \%$ (see Table 3). The NFE of the NMD method is defined as

$$
N F E=\left|\frac{\left(A_{\mathrm{m}}\right)_{\text {fitted }}-A_{\mathrm{m}}}{A_{\mathrm{m}}}\right| \times 100 \%,
$$

in which $\left(A_{\mathrm{m}}\right)_{\text {fitted }}$ denotes the fitted maximum oscillation using the NMD method. Based on the response amplitudes shown in Fig. 17 and the fact that the free surface elevation inside the harbor is a linear superposition of different resonant modes, the total wave energy inside the harbor can be accurately calculated by the following formula 


$$
E=\sum_{i=1}^{20} \frac{1}{2} A_{i}^{2},
$$

in which $A_{i}(i=1,2, \ldots, 20)$ denotes the response amplitude of the $i$-th resonant mode. Fig. 18 presents the total wave energy inside the harbor for the cases shown in Fig. 17. The total wave energy inside the harbor used in Group $\mathrm{E}$ has the minimal value for both $A_{0}=0.02 \mathrm{~m}$ and $A_{0}=0.1 \mathrm{~m}$, which indicates that the largest wave energy reflection occurs inside this harbor. This finding can reasonably explain why in Fig. 12, the numerical result of the wave height evolution of the incident wave in Group E is slightly less than the analytical prediction of Green's law. Meanwhile, the total wave energy inside the harbor does not monotonically increase with the mean water depth inside the harbor, which indicates that the total wave energy inside the harbor depends on both the mean water depth and the bottom profile inside the harbor.
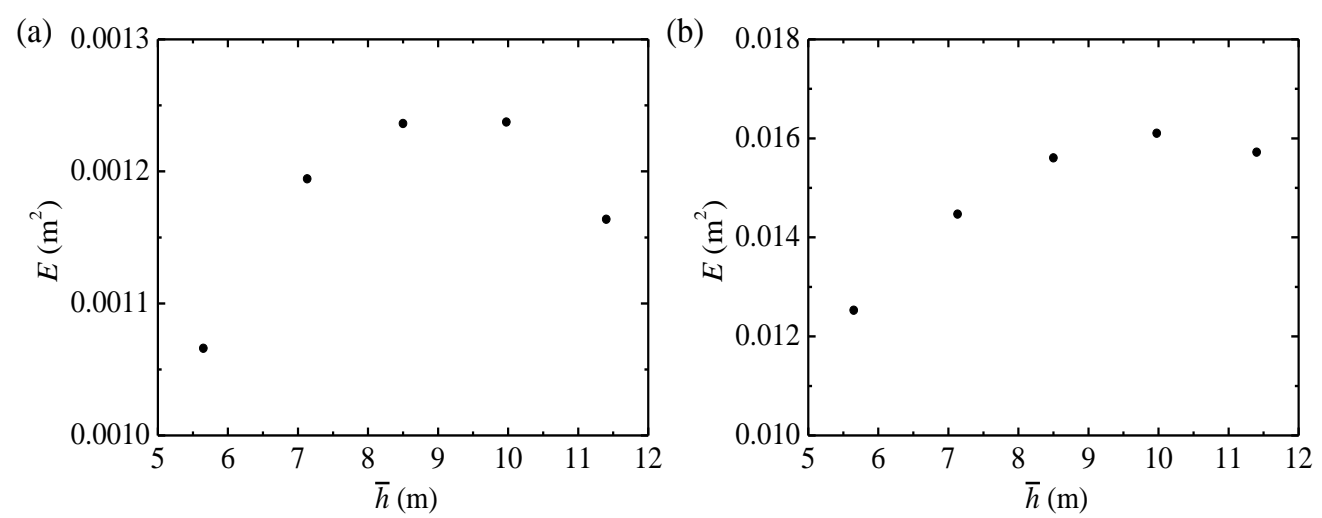

Fig. 18. Total wave energy inside the harbors used in Groups A-E for (a) $A_{0}=0.02 \mathrm{~m}$ and (b) $A_{0}=0.1 \mathrm{~m}$
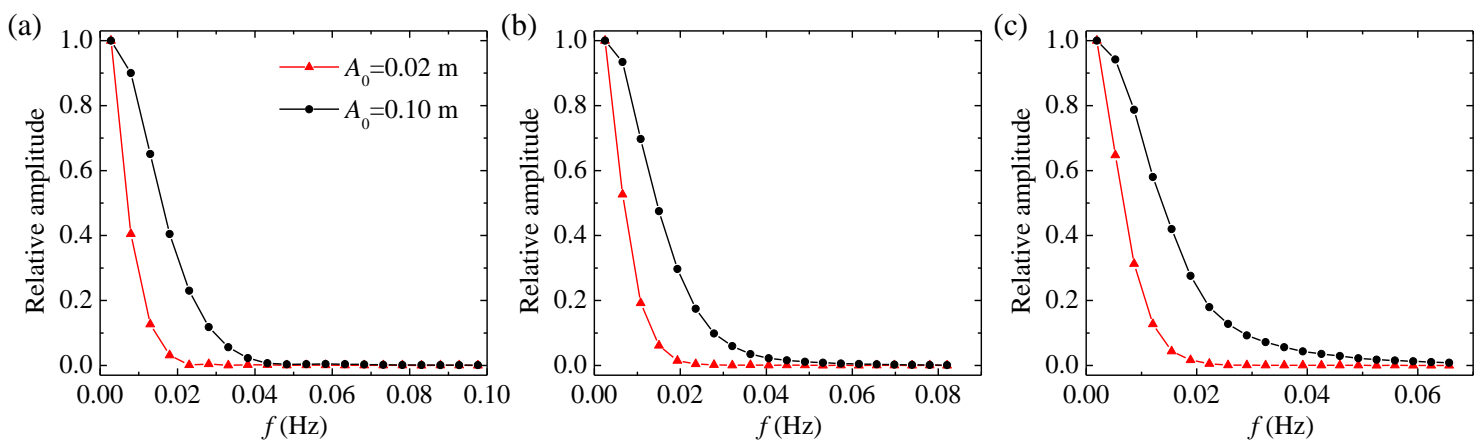

Fig. 19. Relative amplitude distributions of the lowest twenty resonant modes in the numerical experiments with $A_{0}=0.02 \mathrm{~m}$ and $0.10 \mathrm{~m}$ for (a) Group A, (b) Group C and (c) Group E.

In this article, the "relative amplitude" in each case is defined as the ratio of the response amplitude of each resonant mode to that of the first mode. Fig. 19 shows the relative amplitude 
distributions of the lowest twenty resonant modes in the numerical experiments with the incident solitary wave height $A_{0}=0.02 \mathrm{~m}$ and $0.10 \mathrm{~m}$ for Groups A, C and E. It can be found that the relative amplitude distribution inside the harbor of each group is greatly affected by the incident solitary wave height. In the cases with $A_{0}=0.02 \mathrm{~m}$, the wave energy inside the harbor is mainly concentrated in the lowest four modes. When the incident solitary wave height increases, the wave energy tends to spread over a larger range of frequency. In the cases with $A_{0}=0.10 \mathrm{~m}$, the wave energy is concentrated in the more resonant modes (mainly in the lowest eight modes).
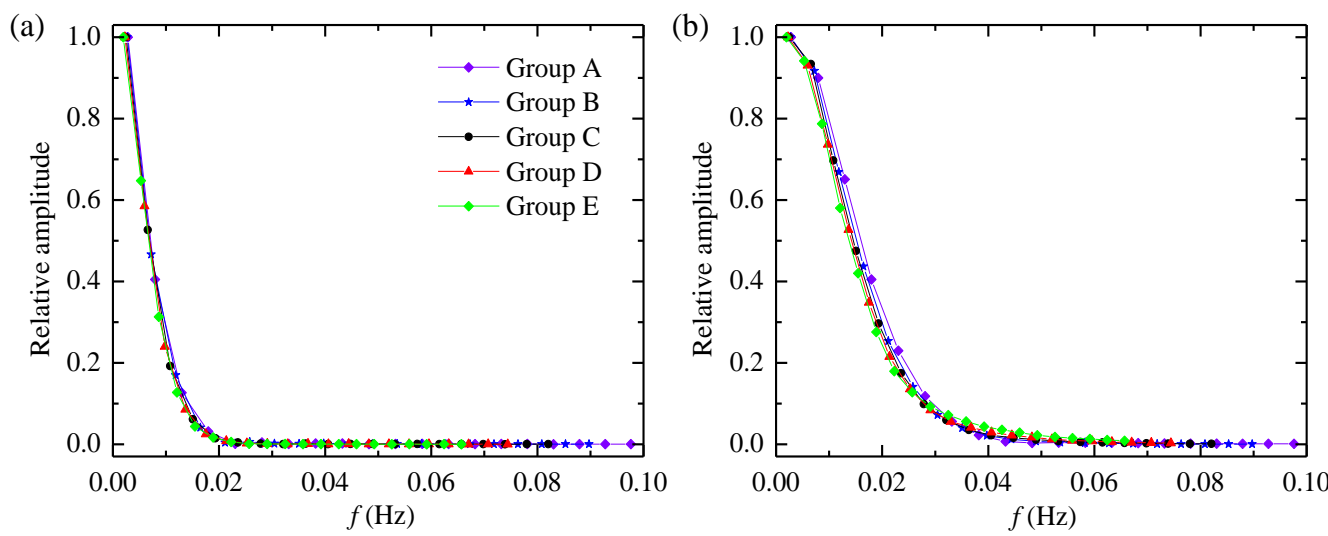

Fig. 20. Comparison of the relative amplitude distribution of the lowest twenty resonant modes when a solitary wave with a given height induces oscillations inside harbors with different bottom profiles: (a) $A_{0}=0.02 \mathrm{~m}$ and (b) $A_{0}=0.10 \mathrm{~m}$

To investigate the effect of different bottom profiles on the relative wave energy distribution, Fig. 20 shows a comparison of the relative amplitude distribution of the lowest twenty resonant modes for a solitary wave with a given height inducing oscillations inside the harbors with different bottom profiles. For all the cases with $A_{0}=0.02 \mathrm{~m}$ or $A_{0}=0.10 \mathrm{~m}$, the effect of different bottom profiles on the relative amplitude distribution seems to be negligible. Because the wave energy is proportional to the square of the wave height, it can be concluded that the relative wave energy distribution inside the harbor is not sensitive to the bottom profile.

\section{Conclusions}

In this paper, harbor oscillations induced by normal-incident solitary waves with different heights inside elongated rectangular harbors with different bottom profiles are simulated using the FUNWAVE 2.0 model. The influences of different incident solitary wave heights and different 
bottom profiles on the wave height evolution, the oscillation amplification, the total wave energy and the relative wave energy distribution inside the harbor are investigated systematically. Different from Dong et al. (2010a) and Gao et al. (2016b), the wave conditions inside the harbor are extended to consider strong wave nonlinearity close to wave breaking. The results of this study will improve the understanding of harbor oscillations induced by the transient nonlinear long waves, such as tsunamis.

The following conclusions can be drawn from the results of the present study:

1. When the incident solitary wave height is small, the height of the incident wave inside the harbor is always larger than that of the reflected wave from the backwall; while as the incident solitary wave height increases, the former gradually becomes smaller than the latter, and the less the mean water depth is, the more obvious the tendency becomes. No matter whether the wave nonlinearity inside the harbor is weak or strong, the height evolution of the incident wave during the shoaling process inside harbors with different bottom profiles coincides well with Green's law, except in the area where the incident and the reflected waves interact with each other near the backwall.

2. When the wave nonlinearity inside the harbor is relatively weak, the maximum oscillation inside the harbor can be regarded as increasing linearly with the incident solitary wave height. However, as the wave nonlinearity becomes strong, the amplification factor increases gradually with the wave nonlinearity. The smaller mean water depth inside the harbor leads to the larger increasing rate of the amplification factor. In the range of the wave nonlinearity studied in this paper, Eq. (13) with the terms of $A_{0}{ }^{2}$ and $A_{0}{ }^{3}$ seems to accurately describe the amplification of the transient long waves inside the harbor.

3. The total wave energy trapped in the harbor does not monotonically change with respect to the mean water depth. It depends on both the mean water depth and the bottom profile. The relative wave energy distribution inside the harbor is greatly affected by the incident solitary wave height. The incident solitary wave with larger height leads to more uniform relative wave energy distribution inside the harbor. However, the effect of different bottom profiles on the relative wave energy distribution is negligible.

Finally, we reaffirm here that these conclusions are only valid for the elongated harbor and when the wave nonlinearity inside the harbor is less than 0.74 . The energy dissipation due to the 
flow separation near the entrance and wave breaking may markedly change the wave climate inside the harbor. These processes will be considered in future studies.

\section{Acknowledgments}

This work was financially supported by the National Natural Science Foundation of China (Grant nos. 11172058, 51221961, 51422901), the Jiangsu Key Laboratory of Advanced Design and Manufacturing Technology for Ship (Grant no. CJ1504) and the Jiangsu Key Laboratory of Coast Ocean Resources Development and Environment Security (Grant no. JSCE201508).

\section{References}

Bellotti, G., 2007. Transient response of harbours to long waves under resonant conditions. Coastal Engineering 54 (9), 680-693.

Bellotti, G., Briganti, R., Beltrami, G.M., 2012. The combined role of bay and shelf modes in tsunamis amplification along the coast. Journal of Geophysical Research 117, C08027, doi: 10.1029/2012JC008061.

Bowers, E.C., 1977. Harbour resonance due to set-down beneath wave groups. Journal of Fluid Mechanics 79, 71-92.

Bruno, D., Serio, F.D., Mossa, M., 2009. The FUNWAVE model application and its validation using laboratory data. Coastal Engineering 56 (7), 773-787.

Byatt-Smith, J.G.B., 1971. An integral equation for unsteady surface waves and a comment on the Boussinesq equation. Journal of Fluid Mechanics 49 (4), 625-633.

Camfield, F., Street, R., 1969. Shoaling of solitary waves on small slopes. J. Waterways and Harbours Div. 95, 1-22.

Chawla, A., Kirby, J.T., 2000. A source function method for generation of waves on currents in Boussinesq models. Applied Ocean Research 22 (2), 75-83.

De Jong, M.P.C., Battjes, J.A., 2004. Seiche characteristics of Rotterdam Harbour. Coastal Engineering 51 (5-6), 373-386

Dong, G., Gao, J., Ma, X., Wang, G., Ma, Y., 2013. Numerical study of low-frequency waves during harbor resonance. Ocean Engineering 68, 38-46.

Dong, G., Wang, G., Ma, X., Ma, Y., 2010a. Harbor resonance induced by subaerial 
landslide-generated impact waves. Ocean Engineering 37 (10), 927-934.

Dong, G., Wang, G., Ma, X., Ma, Y., 2010b. Numerical study of transient nonlinear harbor resonance. Science China-Technological Sciences 53, 558-565.

Fabrikant, A.L., 1995. Harbor oscillations generated by shear flow. Journal of Fluid Mechanics 282, 203-217.

Gao, J., Ji, C., Gaidai, O., Liu, Y., 2016a. Numerical study of infragravity waves amplification during harbor resonance. Ocean Engineering 116, 90-100.

Gao, J., Ma, X., Dong, G., Wang, G., Ma, Y., 2015. Improvements on the normal mode decomposition method used in harbor resonance. Proceedings of the Institution of Mechanical Engineers, Part M: Journal of Engineering for the Maritime Environment 229 (4), $397-410$.

Gao, J., Ma, X., Dong, G., Wang, G., Ma, Y., 2016b. Numerical study of transient harbor resonance induced by solitary waves. Proceedings of the Institution of Mechanical Engineers, Part M: Journal of Engineering for the Maritime Environment 230 (1), 163-176.

Goring, D.G., 1978. Tsunamis - the propagation of long waves onto a shelf. , Ph.D. dissertation, W. M. Keck Laboratory of Hydraulics and Water Resources, California Institute of Technology, Pasadena, California.

Ippen, A.T., Goda, Y., 1963. Wave Induced Oscillations in Harbors: The Solution for a Rectangular Harbor Connected to The Open Sea. Hydrodynamics Laboratory, Massachusettes Institute of Technology, Washington, D. C.

Kirby, J.T., Long, W., Shi, F., 2003. Funwave 2.0 Fully Nonlinear Boussinesq Wave Model On Curvilinear Coordinates. Center for Applied Coastal Research, Dept. of Civil \& Environmental Engineering, University of Delaware, Newark.

López, M., Iglesias, G., 2014. Long wave effects on a vessel at berth. Applied Ocean Research 47, 63-72.

Lee, J.J., 1971. Wave induced oscillations in harbors of arbitrary geometry. Journal of Fluid Mechanics 45, 375-394.

Lepelletier, T.G., 1980. Tsunamis-harbor oscillations induced by nonlinear transient long waves. Report No. KH-R-41, W. M. Keck Laboratory of Hydraulics and Water Resources, California Institute of Technology, Pasadena, California. 
Lepelletier, T.G., Raichlen, F., 1987. Harbor oscillations induced by nonlinear transient long waves. Journal of Waterway, Port, Coastal and Ocean Engineering 113 (4), 381-400.

Li, Y., 2000. Tsunamis : non-breaking and breaking solitary wave run-up. Ph.D. dissertation, W. M. Keck Laboratory of Hydraulics and Water Resources, California Institute of Technology, Pasadena, California.

Liu, P.L.-F., Cho, Y.-S., Briggs, M.J., Kanoglu, U., Synolakis, C.E., 1995. Runup of solitary waves on a circular island. Journal of Fluid Mechanics 302, 259-285.

McCowan, J., 1894. On the highest wave of permanent type. Philosophical Magazine 38 (Series 5), 351-358.

Mei, C.C., 1983. The Applied Dynamics of Ocean Surface Waves. Wiley, New York.

Mei, C.C., Agnon, Y., 1989. Long-period oscillations in a harbour induced by incident short waves. Journal of Fluid Mechanics 208, 595-608.

Miles, J., Munk, W., 1961. Harbor paradox. Journal of the Waterways and Harbors Division 87, $111-132$.

Okihiro, M., Guza, R.T., 1996. Observations of seiche forcing and amplification in three small harbors. Journal of Waterway, Port, Coastal and Ocean Engineering 122 (5), 232-238.

Olsen, K., Hwang, L.-S., 1971. Oscillations in a bay of arbitrary shape and variable depth. Journal of Geophysical Research 76 (21), 5048-5064.

Pattiaratchi, C.B., Wijeratne, E.M.S., 2009. Tide gauge observations of 2004-2007 Indian Ocean tsunamis from Sri Lanka and Western Australia. Pure and Applied Geophysics 166 (1), 233-258.

Rabinovich, A.B., 2009. Seiches and harbor oscillations, in: Kim, Y. (Ed.), Handbook of Coastal and Ocean Engineering. World Scientific Publishing, Singapore, pp. 193-236.

Raichlen, F., Naheer, E., 1976. Wave induced oscillations of harbors with variable depth, Proceedings of 15th International Conference on Coastal Engineering, Honolulu, pp. $3536-3556$

Rogers, S.R., Mei, C.C., 1978. Nonlinear resonant excitation of a long and narrow bay. Journal of Fluid Mechanics 88 (1), 161-180.

Sobey, R.J., 2006. Normal mode decomposition for identification of storm tide and tsunami hazard. Coastal Engineering 53, 289-301. 
Synolakis, C.E., 1987. The runup of solitary waves. Journal of Fluid Mechanics 185, 523-545.

Uzaki, K.-i., Matsunaga, N., Nishii, Y., Ikehata, Y., 2010. Cause and countermeasure of long-period oscillations of moored ships and the quantification of surge and heave amplitudes. Ocean Engineering 37, 155-163.

Vanoni, V.A., Carr, J.H., 1950. Harbor surging, Proceedings of the 1st International Conference on Coastal Engineering, Long Beach, pp. 60-68.

Wang, G., Dong, G., Perlin, M., Ma, X., Ma, Y., 2011a. An analytic investigation of oscillations within a harbor of constant slope. Ocean Engineering 38, 479-486.

Wang, G., Dong, G., Perlin, M., Ma, X., Ma, Y., 2011b. Numerical investigation of oscillations within a harbor of constant slope induced by seafloor movements. Ocean Engineering 38 (17-18), 2151-2161.

Wang, G., Zheng, J., Liang, Q., Zhang, W., Huang, C., 2015. Theoretical analysis of harbor resonance in harbor with an exponential bottom profile. China Ocean Engineering 29 (6), 821-834.

Wang, G., Zheng, J., Liang, Q., Zheng, Y., 2014. Analytical solutions for oscillations in a harbor with a hyperbolic-cosine squared bottom. Ocean Engineering 83, 16-23.

Wu, J.K., Liu, P.L.-F., 1990. Harbour excitations by incident wave groups. Journal of Fluid Mechanics 217, 595-613.

Zhao, X., Wang, B., Liu, H., 2012. Characteristics of tsunami motion and energy budget during runup and rundown processes over a plane beach. Physics of Fluids 24 (6), 062107. 